\title{
Gene Expression Profiles for Genotoxic Effects of Silica-Free and Silica-Coated Cobalt Ferrite Nanoparticles
}

\author{
Do Won Hwang ${ }^{1,2}$, Dong Soo Lee ${ }^{1,3}$, and Soonhag Kim ${ }^{4}$ \\ ${ }^{1}$ Department of Nuclear Medicine, Seoul National University College of Medicine, Seoul, Korea; ${ }^{2}$ Institute of Radiation Medicine, \\ Medical Research Center, Seoul, Korea; ${ }^{3}$ WCU Department of Molecular Medicine and Biopharmaceutical Science, Graduate School \\ of Convergence Science and Technology, Seoul, Korea; and ${ }^{4}$ Laboratory of Molecular Imaging, Department of Biomedical Science, \\ College of Life Science, CHA Stem Cell Institute, CHA University, Seoul, Korea
}

\begin{abstract}
Nanomaterials have been widely evaluated for potential use as efficient delivery carriers for cancer diagnosis and therapy. To translate these nanomaterials to the clinic, their safety needs to be verified, particularly in terms of genotoxicity and cytotoxicity. We investigated changes in gene expression profiles influenced by silica-coated cobalt ferrite magnetic-fluorescence nanoparticles and silica-free cobalt ferrite magnetic-core nanoparticles in vivo and in vitro. Methods: ${ }^{68} \mathrm{Ga}$-labeled cobalt ferrite nanoparticles produced by synthesis of 2-(p-isothio-cyanatobenzyl)-1,4,7-triazacyclonane-1,4,7-triacetic acid chelator were established after labeling efficiency had been validated through a thin-layer chromatography method. The expression of genes associated with the stress and toxicity pathways was verified by a commercially available polymerase chain reaction array kit. Results: In comparison with magnetic-fluorescence nanoparticles, magnetic-core nanoparticles revealed severe cytotoxic effects at various doses and treatment times as determined by the 3-(4,5-dimethylthiazol-2-yl)2,5-diphenyltetrazolium bromide assay. Whole-body small-animal PET and biodistribution studies, including transmission electron microscope analysis, showed that tail-vein injection of magneticcore or magnetic-fluorescence nanoparticles exhibited substantial liver accumulation. Real-time polymerase chain reaction array using 52 genes related to cellular toxicity demonstrated that 17 genes from the magnetic-core-treated liver samples were significantly affected, mostly in relation to DNA damage or repair and to oxidative or metabolic stress. The magnetic-fluorescence-treated liver samples showed gene expression approximately $90 \%$ similar to that of untreated liver samples. Conclusion: We compared a variety of gene expression profiles in mice injected with magnetic-fluorescence or magnetic-core nanoparticles. This study of gene expression profiles affected by nanotoxicity provides critical information for the clinical use of silica-coated cobalt ferrite.
\end{abstract}

Key Words: genotoxicity; cobalt ferrite nanoparticle; microPET imaging; silica-coated nanoparticle

J Nucl Med 2012; 53:106-112

DOI: 10.2967/jnumed.111.088443

\footnotetext{
Received Jan. 25, 2011; revision accepted Jul. 5, 2011.

For correspondence or reprints contact: Soonhag Kim, Laboratory of Molecular Imaging Department of Biomedical Science, College of Life Science, CHA Stem Cell Institute, CHA University, 605-21 Yoeksam 1-dong, Gangnam-gu, Seoul, Korea.

E-mail: kimsoonhag@empal.com

Published online Dec. 6, 2011

COPYRIGHT @ 2012 by the Society of Nuclear Medicine, Inc.
}

I n recent years, biologic and biomedical applications in health care have rapidly progressed, and engineered nanoscale materials have attracted much attention because of their potential for use as efficient drug delivery carriers for diagnostic and therapeutic applications (1-5).

However, amid the rapid advances in the application of nanotechnology to human medicine for theragnosis, safety issues have been considered one of the most critical problems to be solved. Thus far, adverse effects of nanomaterials on biologic systems have been proven through a variety of toxicologic experiments. Most studies related to nanotoxicity have been at the cellular level, examining cell viability to evaluate the usefulness of nanoparticles in clinical fields $(6-11)$. The acute toxicity of polyethylene glycol (PEG)-tethered gold nanoparticles has been verified in vivo by measurement of inflammation- and apoptosisrelated gene expression levels in liver tissues (12). However, to better understand nanotoxicity, it is important to use simple in vivo and in vitro methods to investigate the dynamics of many different gene expressions affected by nanomaterials. Unlike previous studies, which investigated only a few gene expression patterns in vivo, the current study examined changes in numerous gene expression patterns in vivo and in vitro after nanomaterial exposure, using a simple PCR array method of evaluating toxicologic effects.

A variety of methods for testing genotoxicity, such as the comet assay or micronucleus test, has generally been used for detecting potential DNA damage (13). Also, gene-expression analysis is one important factor in genotoxicity tests, with real-time reverse transcription (RT) polymerase chain reaction (PCR) becoming the method of choice for high-throughput and accurate expression profiling of selected genes under single-experiment conditions. Quantitative real-time RT PCR analysis provides substantial microarraylike information on nanotoxicity-related changes in a host of gene expressions, which can be assayed simultaneously with high sensitivity and specificity, offering multiple gene expression profiling.

Previous studies have demonstrated - not on the basis of gene expression but of cellular viability-that silica-coated cobalt ferrite nanoparticles are safe. A variety of cytotoxicity 
tests have shown not only normal histopathologic data in magnetic-fluorescence-induced mouse organs but also no significant chromatid exchange or breakthrough chromosomal aberrations (14). We compared the effect of silicacoated cobalt ferrite magnetic-fluorescence nanoparticles with that of silica-free cobalt ferrite magnetic-core nanoparticles on gene expression of cellular stress- and toxicityrelated messenger RNA, with focus on the potential genotoxic and cytotoxic effects of the 2 types of nanoparticles in vitro and in vivo. We examined the gene expression profiles for oxidative or metabolic stress, apoptosis signaling, growth arrest, DNA damage, and inflammation-related genes under a toxic environment, such as silica-free and -coated nanoparticle-treated mouse liver tissue and cultured hepatocarcinoma cells, after liver accumulation was clearly visualized by a PET imager using ${ }^{68} \mathrm{Ga}$-labeled nanoparticles.

\section{MATERIALS AND METHODS}

\section{${ }^{68}$ Ga-Labeled Nanoparticle Synthesis}

Magnetic-fluorescence nanoparticles are composed of a cobalt ferrite $\left(\mathrm{CoFe}_{2} \mathrm{O}_{4}\right)$ magnetic core and rhodamine $\mathrm{B}$ isothiocyanate (excitation/emission, $555 / 578 \mathrm{~nm}$ ) coated with a silica shell, which is linked with PEG $\left(1.1 \times 10^{4} /\right.$ nanoparticle $)$ and amine moieties $\left(3.9 \times 10^{4} /\right.$ nanoparticle $)$ on the surface. Magnetic-core nanoparticles contain only cobalt ferrite without a silica coating. The basic core nanoparticles were commercially designed by Biterials, and the procedure for basic core synthesis was as previously described $(5,15)$. Briefly, cobalt ferrite magnetics were stabilized using polyvinyl pyrrolidone (Sigma) solution. Trimethoxysilane modified by rhodamine B isothiocyanate was injected into the prepared polyvinyl pyrrolidone-stabilized magnetic nanoparticles. To yield rhodamine B isothiocyanate-containing silica magnetic nanoparticles ( $4 \times 10^{3} /$ nanoparticle), polymerization was performed by adding ammonia solution. The magnetic-core nanoparticles (size, $32.6 \pm$ $4.5 \mathrm{~nm}$ ) also contain PEG and an amine group on the surface and are supplied as a powder and resuspended with phosphate-buffered saline for use in a biologic study. Before synthesis of the magneticcore or magnetic-fluorescence nanoparticles with $p$-SCN-Bn-NOTA (2-( $p$-isothio-cyanatobenzyl)-1,4,7-triazacyclonane-1,4,7-triacetic acid), sonication was performed for $10 \mathrm{~min}$ to produce a homogeneous monodispersion of these nanoparticles. The reaction was performed in $\mathrm{NaHCO}_{3}$ buffer solution ( $\mathrm{pH}$ 9). The synthesis between amine-terminated magnetic-core (or magnetic-fluorescence) nanoparticles was incubated with $p$-SCN-Bn-NOTA (bifunctional chelator) under mild stirring at $4^{\circ} \mathrm{C}$ overnight. The synthesized magnetic-core or magnetic-fluorescence nanoparticles dispersed in $\mathrm{NaHCO}_{3}$ buffer were collected by high centrifugation at $15,000 \mathrm{rpm}$ for $10 \mathrm{~min}$, followed by washing with phosphatebuffered saline. As a next step for labeling with ${ }^{68} \mathrm{Ga},{ }^{68} \mathrm{GaCl}_{3}$ was eluted using $0.1 \mathrm{M} \mathrm{HCl}(432.9 \mathrm{MBq}[11.7 \mathrm{mCi}] / 4 \mathrm{~mL})$ in a ${ }^{68} \mathrm{Ge} /{ }^{68} \mathrm{Ga}$ generator. $p$-SCN-Bn-NOTA-synthesized magnetic-core or magneticfluorescence nanoparticles were labeled with eluted ${ }^{68} \mathrm{GaCl}_{3}$ with $0.2 \mathrm{M} \mathrm{Na}_{2} \mathrm{HPO}_{4} / \mathrm{NaH}_{2} \mathrm{PO}_{4}$ (Sigma) buffer solution at $\mathrm{pH}$ 6.5. The mixture was incubated at room temperature for $1 \mathrm{~h}(16) .{ }^{68} \mathrm{Ga}$-labeled magnetic-core or magnetic-fluorescence nanoparticles were prepared by high-speed centrifugation.

\section{Transmission Electron Microscope (TEM) Study}

Liver samples treated with magnetic-core or magnetic-fluorescence nanoparticles were fixed with a $4 \%$ paraformaldehyde solution and dehydrated on graded concentrations of alcohol. Negatively stained specimens were placed on polyvinyl formal resin carbon-coated grids, and TEM studies were conducted using a JEM-1010 (Jeol Korea Ltd.) at $80 \mathrm{kV}$. Electron microscopic digital images were obtained using a cooled charge-coupleddevice camera (Gatan, Inc.).

\section{3-(4,5-Dimethylthiazol-2-yl)-2,5-Diphenyltetrazolium Bromide (MTT) Assay}

Human hepatoma Hep3B cells $\left(2 \times 10^{4}\right)$ were plated into 96well plates with $200 \mu \mathrm{L}$ of culture medium. After $24 \mathrm{~h}$, each dose of nanoparticle was resuspended in phosphate-buffered saline and used to treat the cells for $24 \mathrm{~h}$. The cells were exposed to $20 \mu \mathrm{L}$ of MTT solution (1 mg/mL) and incubated for $4 \mathrm{~h}$. Dimethyl sulfoxide $(200 \mu \mathrm{L})$ was added to solubilize formazan crystals. Optical density was determined using an ELISA reader at 5,600-nm wavelength. All samples were evaluated in triplicate. Time-dependent tests of cellular toxicity were performed for up to $3 \mathrm{~d}$.

\section{Confocal Microscopy of Liver}

Frozen liver tissue isolated from mice injected with each nanoparticle was prepared and sectioned at a thickness of $5 \mu \mathrm{m}$. The tissue slices were placed on glass slides and mounted with solution containing DAPI (Vector Laboratories, Inc.). Rhodamine fluorescence images were obtained using a confocal microscope (model LSM 510; Zeiss).

\section{Quantitative Real-Time PCR Array}

Nanoparticle-injected liver tissues and nanoparticle-treated cells were prepared for RNA purification. Isolated liver tissues were frozen in liquid nitrogen and homogenized, and 10-30 mg of tissue were harvested with TRIzol reagent (Invitrogen). RT of $1 \mu \mathrm{g}$ of total RNA was performed using SuperScript II (Invitrogen) reverse transcriptase to yield complementary DNA for each sample. A kit (RT ${ }^{2}$ Profiler PCR array system; SABiosciences) was used to examine multiple gene expressions related to the stress and toxicity pathways. The synthesized complementary DNA samples were mixed with a master mixture for preparation of quantitative realtime PCR array. Plates (96-well) containing all reagents were briefly centrifuged, and real-time quantitative PCR was performed using an ABI Prism 7900 HT detector (Applied Biosystems) under the following conditions: $50^{\circ} \mathrm{C}$ for $2 \mathrm{~min}, 95^{\circ} \mathrm{C}$ for $10 \mathrm{~min}$, and 40 cycles of $95^{\circ} \mathrm{C}$ for $15 \mathrm{~s}$ and $60^{\circ} \mathrm{C}$ for $1 \mathrm{~min}$. Threshold cycle values were normalized to the glyceraldehyde-3-phosphate dehydrogenase (GAPDH) gene, and the expression data were represented as fold ratio values. Threshold cycle values determined by realtime RT PCR were quantitatively calculated as follows:

$$
\begin{aligned}
& \Delta \mathrm{TC} \text { value }=\text { each sample } \mathrm{TC} \text { value }-\mathrm{GAPDH} \text { TC value }, \\
& \Delta \Delta \mathrm{TC} \text { value }=\Delta \mathrm{TC} \text { value }- \text { lowest } \mathrm{TC} \text { value, }
\end{aligned}
$$$$
\text { Acquired value }=2^{-\Delta \Delta \mathrm{TC}} \text {, }
$$

where TC is threshold cycle.

\section{Small-Animal PET and Biodistribution of ${ }^{68}$ Ga-Labeled Nanoparticles}

Animal handling and experimentation were performed according to the guidelines of the Institutional Animal Care and Use Committee of Seoul National University Hospital. A ${ }^{68}$ Ga-labeled 
magnetic-core or magnetic-fluorescence nanoparticle suspension $(500 \mu \mathrm{g})$ was administered by tail vein injection to 6-wk-old BALB/c- $\nu$-mice ( 3 mice per group), which were then anesthetized with $1 \%$ isoflurane- $\mathrm{O}_{2}$ air and placed prone under a small-animal PET/CT scanner (eXplore Vista; GE Healthcare). Dynamic PET images were acquired over $10 \mathrm{~min}$ in 6 frames. The mice were sacrificed $1 \mathrm{~h}$ after injection of the nanoparticles; various organs, including the liver, were isolated and weighed; and radioactivity was counted with a $\mathrm{NaI}$ well counter. Biodistribution was represented as a percentage of the injected dose per gram of tissue. The liver-to-muscle ratio was evaluated quantitatively from reconstructed data using Vista software. Regions of interest were manually drawn over the liver and muscle regions for analysis.

\section{Statistical Analysis}

All data are presented as the mean \pm SEM from individual experiments and calculated using the Student $t$ test. Statistical significance was accepted at $P$ values of less than 0.05 .

\section{RESULTS}

\section{In Vitro Cellular Toxicity}

TM-50 PEG and $\mathrm{NH}_{2}$ (silica-free cobalt ferrite magneticcore nanoparticles) were designed for use in the control group by combining an amine group and PEG moieties on the surface area. Magnetic nanoparticles@ $\mathrm{SiO}_{2}$ (rhodamine $\mathrm{B}$ isothiocyanate)-PEG/ $\mathrm{NH}_{2}$ (silica-coated cobalt ferrite magnetic-fluorescence nanoparticles) were previously reported and have been well characterized $(4,5)$. Briefly, the magnetic-fluorescence nanoparticles were synthesized to have a cobalt ferrite core coated with a silica shell containing rhodamine B isothiocyanate (excitation/ emission, 555/578 nm). TEM images showed the magnetic-fluorescence nanoparticles to appear as monodispersed crystalline spheres with a size of approximately $50 \mathrm{~nm}$ (Supplemental Fig. 1A), whereas the magnetic-core nanoparticles showed a polydispersed pattern and an approximate magnetic-core size of $35 \mathrm{~nm}$ (Supplemental Fig. 1B). To compare the cytotoxic effects of the 2 types of nanoparticles at the cellular level, each type was used to treat Hep3B cells. An MTT colorimetric assay of magneticcore-treated cells showed an explicit time-dependent reduction of cell viability. No significant reduction in the viability of magnetic-fluorescence-treated cells was seen until 3 d (Fig. 1A). Also, when various doses of nanoparticles were used to treat the Hep3B cell line, magnetic-core nanoparticles were significantly more cytotoxic than magnetic-fluorescence nanoparticles; a dose-dependent reduction of viability was observed only in the cells treated with magnetic-core nanoparticles (Fig. 1B).

\section{In Vivo Small-Animal PET and Biodistribution}

Because the mononuclear phagocytic system allows nanosized particles to accumulate naturally in liver tissue through opsonization phenomena $(17,18)$, the liver is a good target organ for in vivo nanotoxicity studies. To monitor retention of nanoparticles in the liver noninvasively, a PET approach providing tomographic information with high sensitivity was used. Amine moieties in magnetic-core and magnetic-fluorescence nanoparticles were synthesized with a $p$-SCN-Bn-NOTA chelator and subsequently labeled with ${ }^{68} \mathrm{GaCl}_{3}$. The labeling efficiency of ${ }^{68} \mathrm{Ga}$-labeled magnetic-core and magnetic-fluorescence nanoparticles was checked by thin-layer chromatography. The ${ }^{68} \mathrm{Ga}$-labeled magnetic-fluorescence nanoparticles were tested for stability under serum incubation and showed a stable structure for 2 or $4 \mathrm{~d}$ (Supplemental Fig. 2). Each radiolabeled nanoparticle was administered intravenously to mice, and dynamic small-animal PET images were acquired. ${ }^{68} \mathrm{Ga}-$ labeled magnetic-core-injected mice exhibited radionuclide signals exclusively in liver tissue through the coronal, sagittal, and axial planes (Fig. 2A). Dynamic small-animal PET for quantitative measurements was performed over $10 \mathrm{~min}$ in 6 frames and showed that ${ }^{68} \mathrm{Ga}$ radioactivity gradually decreased through the region-of-interest value

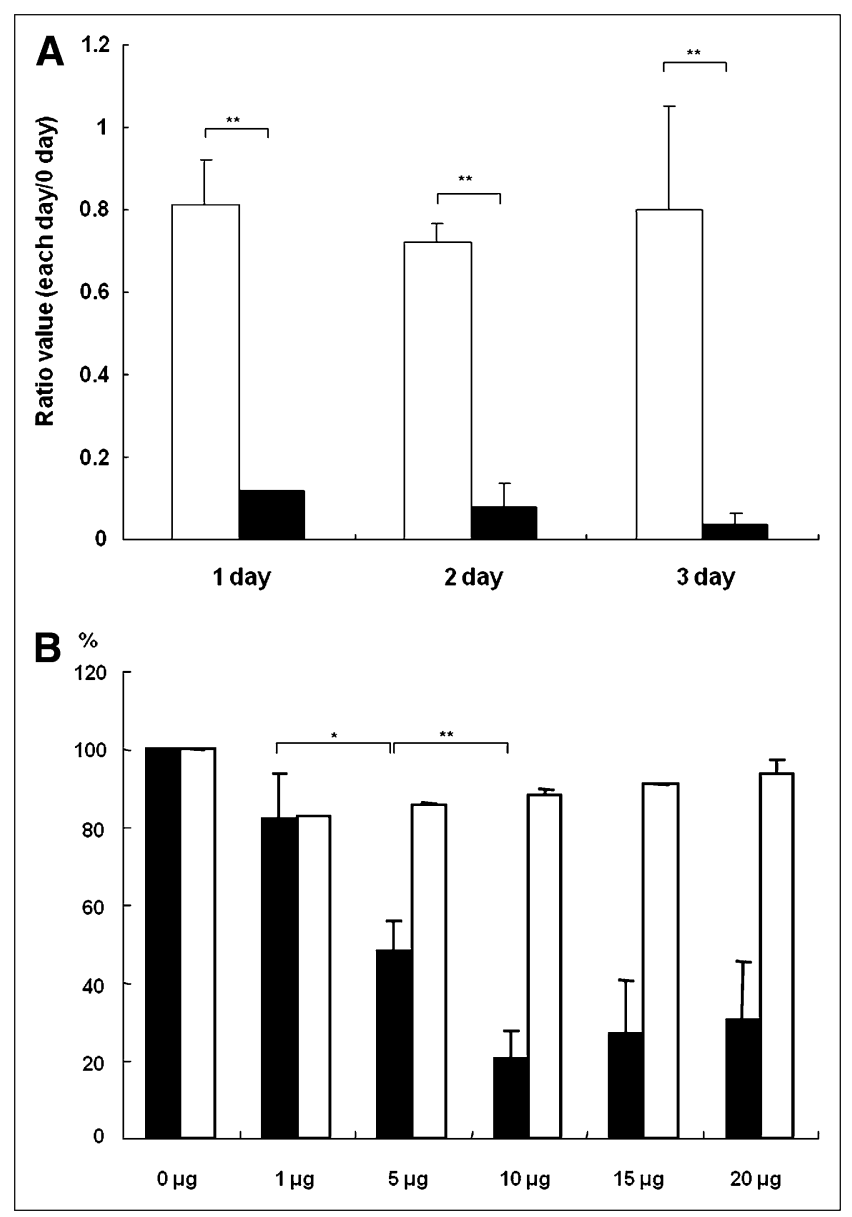

FIGURE 1. In vitro cytotoxic effects of magnetic-core (black bars) and magnetic-fluorescence (white bars) nanoparticles. Twenty micrograms of each type were used to treat Hep3B hepatocarcinoma cell line, and time-dependent (A) and dose-dependent (B) MTT assay was performed. Significant reduction of cell viability was observed $1 \mathrm{~d}$ after treatment with magnetic-core nanoparticles, compared with magnetic-fluorescence nanoparticles. Cell viability gradually decreased until $10-\mu \mathrm{g}$ concentration of magnetic-core nanoparticles was reached. ${ }^{\star} P<0.05$. ${ }^{* \star} P<0.0005$. 
for the liver-to-muscle ratio (Supplemental Fig. 3). One hour after the ${ }^{68} \mathrm{Ga}$-labeled nanoparticles had been administered, biodistribution studies showed an approximately 50-fold higher accumulation of both types of nanoparticles in liver tissue than in muscle (Fig. 2B).

\section{Ex Vivo Validation Study in Liver}

To determine whether the acquired high liver uptake was the result of magnetic-core or magnetic-fluorescence nanoparticles, we performed an ex vivo study on fixed liver tissue injected with both types of ${ }^{68} \mathrm{Ga}$-labeled nanoparticles. Confocal microscope images of mouse liver showed evenly dispersed rhodamine fluorescence signals in the tissue that had been treated with magnetic-fluorescence nanoparticles (Supplemental Fig. 4). As expected, no fluorescence signal was detected in untreated and magneticcore-treated mice. However, TEM revealed an abundance of magnetic-core or magnetic-fluorescence nanoparticles in vesiclelike spaces in the cytoplasm (Supplemental Fig. 5). Hematoxylin and eosin staining of liver sections from each group revealed that nuclei in magnetic-core-treated liver were of irregular shape, compared with the shape of nuclei in untreated and magnetic-fluorescence-treated liver (Supplemental Fig. 6).

\section{Genotoxic Effect of Magnetic-Core Nanoparticles}

We investigated the effect of nanoparticles on multiple gene expression profiles through fluorescence-based realtime RT PCR analysis. The expression levels of 52 genes, which were divided into 7 gene functional groups (oxidative or metabolic stress, heat shock, proliferation and carcinogenesis, growth arrest and senescence, inflammation, DNA damage and repair, and necrosis or apoptosis) as shown in Supplemental Table 1, were examined in vivo and in vitro. Twenty-four hours after magnetic-core or magnetic-fluorescence nanoparticles $(100 \mu \mathrm{g})$ had been used to treat Hep3B cells seeded on 10-cm culture dishes, the total RNA of each sample was isolated using TRIzol reagent, and real-time RT PCR analysis was conducted for each gene set using sequence-specific primers. A housekeeping gene, GAPDH, was used as an internal control. The number of genes that had increased by more than 2-fold in each group was as follows: magnetic-fluorescence vs. untreated, 14 genes in vitro and 2 genes in vivo; magnetic-core vs. magnetic-fluorescence, 22 genes in vitro and 17 genes in vivo; magnetic-core vs. untreated, 27 genes in vitro and 15 genes in vivo. Of the 52 genes, 22 had a 2 -fold higher level of expression in magnetic-core-treated He3B cells than in untreated or magnetic-fluorescence-treated cells (Fig. 3A).
A
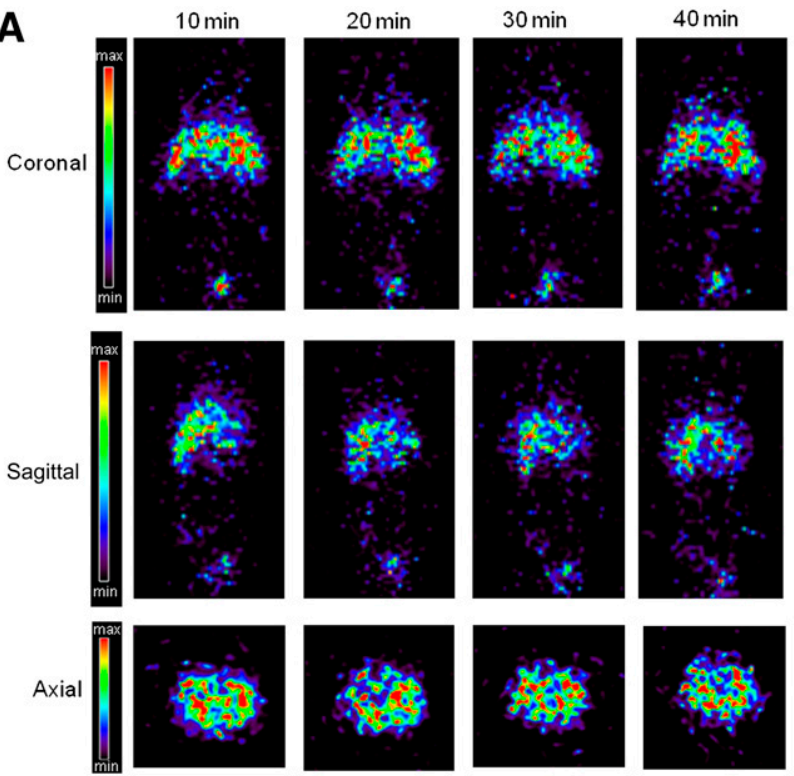

B

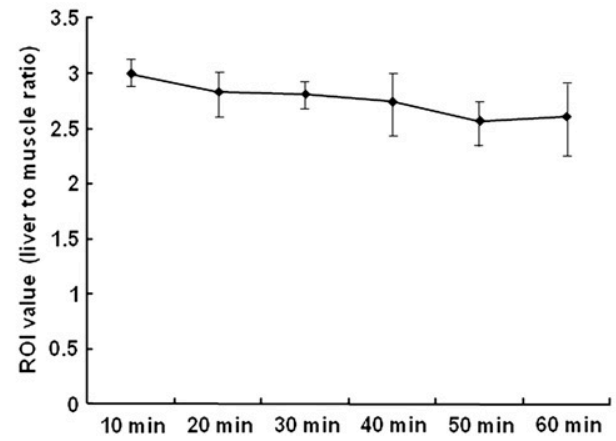

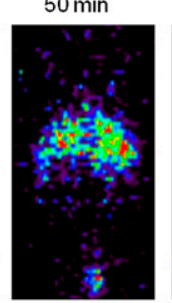
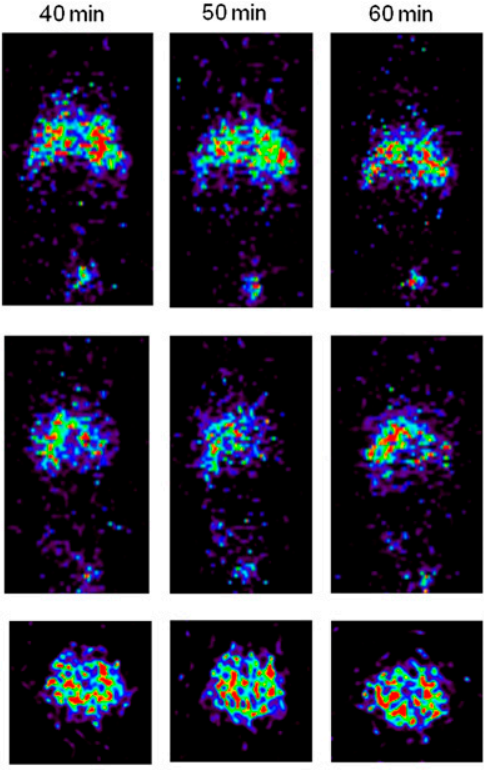
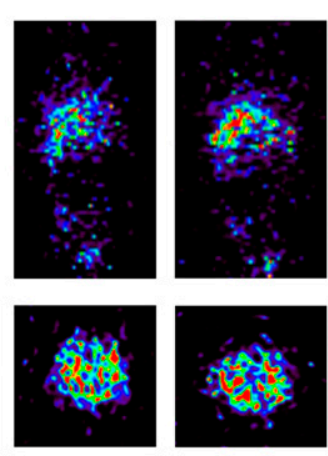

FIGURE 2. Dynamic small-animal PET and biodistribution study. (A) Dynamic PET images (coronal, sagittal, and axial views) showing exclusive accumulation of ${ }^{68} \mathrm{Ga}-\mathrm{la}$ beled magnetic-core nanoparticles in liver, with radioactivity remaining until $60 \mathrm{~min}$. (B) Biodistribution profile for mice injected with ${ }^{68} \mathrm{Ga}-$ labeled magnetic-core (black bars) and magnetic-fluorescence (white bars) nanoparticles. Data are expressed as percentage of injected dose per gram of tissue. 
Among these 22 genes, genes related to oxidative or metabolic stress, including Cyp3a11, as well as DNA damage and repair, including Xrcc2, were highly upregulated, compared with expression of the same genes in magneticfluorescence-treated or untreated cell lines. Five genes (Dnaja1, Gadd45a, Il1a, Lta, and NfKBia) had a relatively similar pattern of expression between the magnetic-coreand magnetic-fluorescence-treated groups (Supplemental Fig. 7A). For the in vivo genotoxic study, liver tissues were isolated $24 \mathrm{~h}$ after injection of magnetic-core or magneticfluorescence nanoparticles. Each total RNA sample was extracted from the magnetic-core-, magnetic-fluorescence-injected, or untreated liver tissue and was reacted on a 96-well PCR array plate containing a master mixture. Of the 52 genes, 17 showed a significantly affected level of expression in silica-free magnetic-core-treated liver, compared with magnetic-fluorescence-treated and untreated liver (Fig. 3B). Seventeen genes were related to DNA damage or repair (Atm, Rad23a, and Rad50), apoptosis (Anxa5 and Fasl), carcinogenesis (Ccnc, Ccnd1, and Ccng1), inflammation (Cxcl10 and Il18), oxidative stress (Gpx2, Gsr, Mt2, Cyp4a10, Cyp4a14, and Por), or growth arrest (Igfbp6). Three genes (apoptosis-related [Tradd], oxidative stress-related [Polr2k], and growth arrest-related [Trp53]) showed a similar pattern of expression in magnetic-core-, magnetic-fluorescence-treated, and untreated liver (Supplemental Fig. 7B).

\section{Gene Expression in Liver}

The in vitro genotoxic results showed a relatively strong correlation $\left(R^{2}=0.68\right)$ of gene expression patterns between the untreated and magnetic-fluorescence-treated groups, compared with the untreated and magnetic-core-treated groups $\left(R^{2}=0.02\right)$ or the magnetic-core-treated and magnetic-fluorescence-treated groups $\left(R^{2}=0.12\right.$; Fig. 4A and Supplemental Fig. 8A). Similarly, a relatively strong correlation $\left(R^{2}=0.89\right)$ was found between the untreated and magneticfluorescence treated groups (Fig. 4B), whereas a weak correlation in each selected gene expression group was found between the magnetic-core and magnetic-fluorescence groups $\left(R^{2}=0.26\right)$ and the untreated and magnetic-core groups $\left(R^{2}=0.24\right)$ (Supplemental Fig. 8B). In vitro and in vivo genotoxic studies showed that genes with at least a 2-fold higher level of expression in the magnetic-core-treated gene group than in the magnetic-fluorescence-treated group were functionally clustered into 7 categories (Supplemental Fig. 9). Both in vitro and in vivo data demonstrated that genes involved in DNA damage or repair and oxidative or
FIGURE 3. Genotoxic effects of magneticcore and magnetic-fluorescence nanoparticles on Hep3B cells and liver tissues. Real-time PCR array was conducted using total RNA samples isolated from 3 groups (untreated [white bars], magnetic-core-treated [hatched bars], and magnetic-fluorescencetreated [black bars]) of Hep3B cells and in vivo liver tissue. (A) Genes significantly affected by magnetic-core nanoparticles when both types of nanoparticles were used to directly treat Hep3B cells. (B) Genes showing significantly enhanced expression in magnetic-core-treated in vivo liver samples when both types of nanoparticles were injected into mice via tail vein.

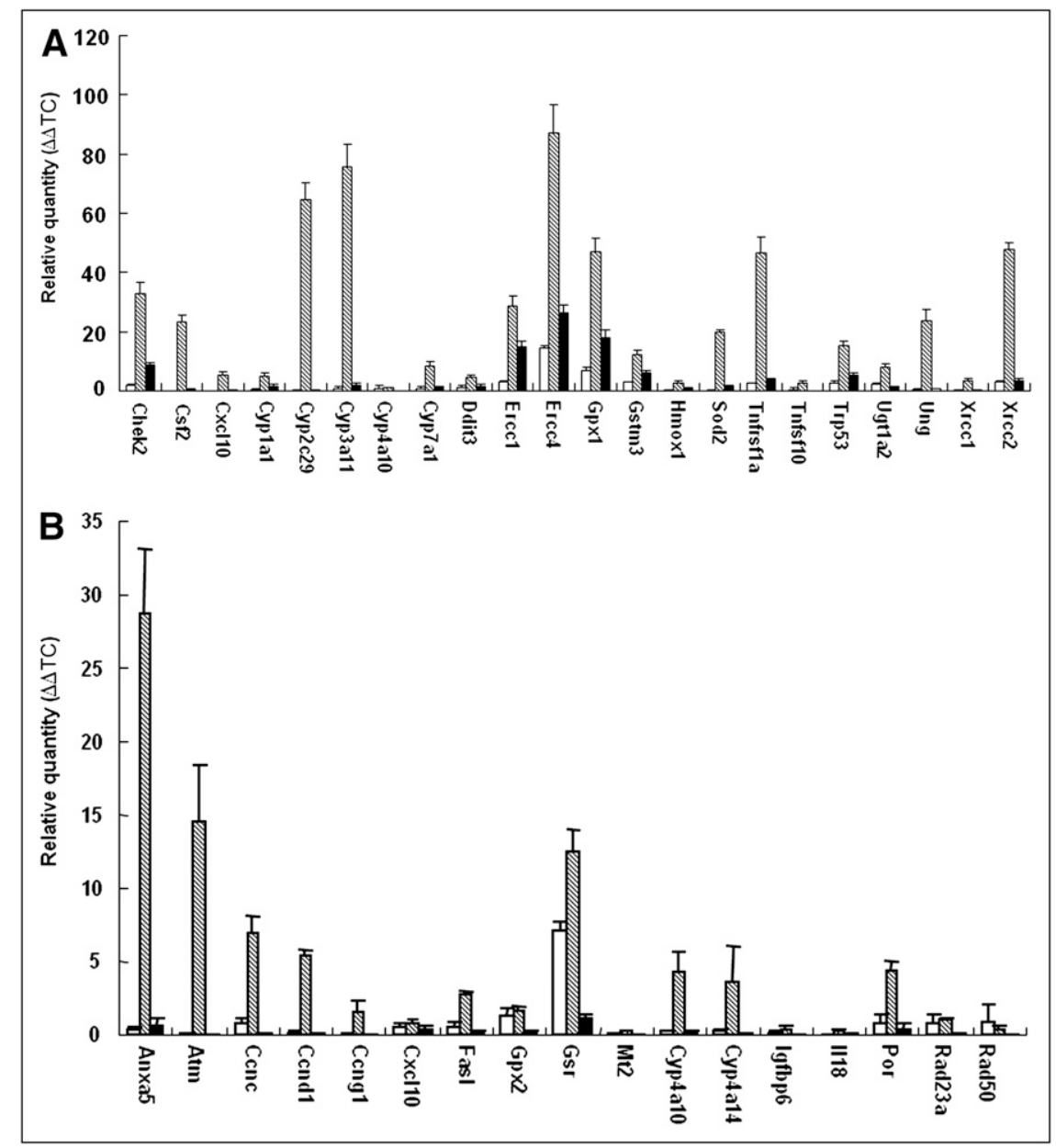


metabolic stress accounted for more than $50 \%$ of the tested genes, indicating that the magnetic-core nanoparticles caused severe DNA damage through cellular stress.

\section{DISCUSSION}

The rapid development of nanotechnology has benefited a variety of fields in biology and medicine. Although much research has progressed from the potential benefits of nanomaterials for chemical sensing, in vitro diagnostics, and in vivo imaging, applications in human health cannot progress without consideration of the potential hazards of nanomaterials. Recently, the biologic impact of nanomaterials on intact tissues and organs has been examined (19-21). However, most toxicologic studies have been performed at the cellular level or provided information about
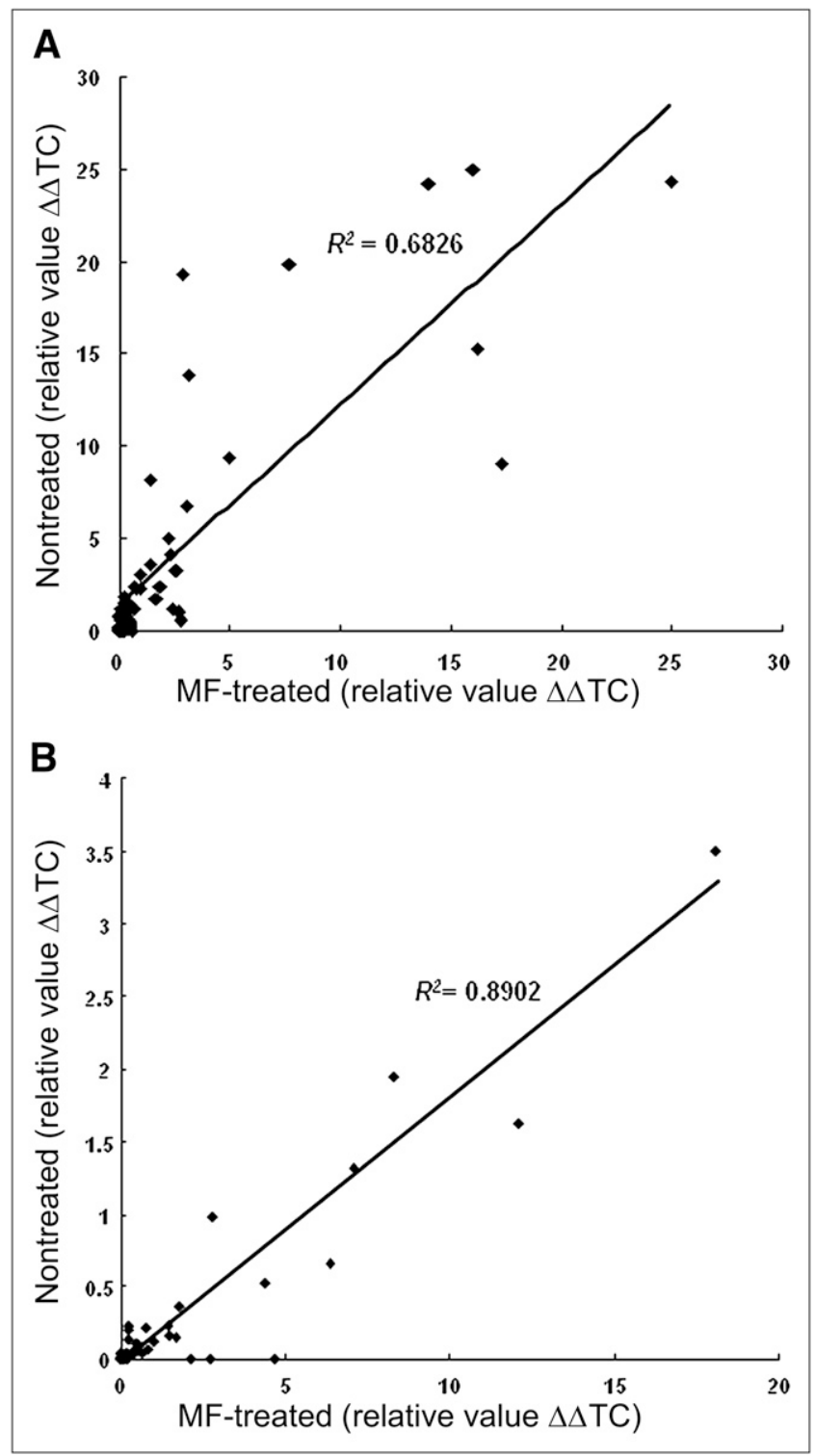

FIGURE 4. Graph showing relatively strong correlation between untreated and magnetic-fluorescence (MF)-treated groups in vitro (A) and in vivo (B). the level of expression of several genes $(12,22,23)$. The toxicologic effects of nanoparticles should be investigated at the genetic level in vivo and in vitro. Thus, we focused on the potential toxicity of nanomaterials for a better understanding of nanotoxicity at the genetic and cellular levels.

Although our in vivo biodistribution study showed substantial liver uptake of magnetic-core and magnetic-fluorescence nanoparticles, a comparison using the MTT assay demonstrated that the magnetic-fluorescence nanoparticles had no significant effect on cell viability but that the magnetic-core nanoparticles showed apparent cytotoxicity. In addition, real-time PCR array revealed that the magneticcore nanoparticles stimulated a significantly increased expression of genes involved in the stress and toxicity signal pathways in vitro and in vivo, whereas magneticfluorescence nanoparticles showed a gene expression pattern similar to that of the untreated group. Magneticcore-treated liver samples showed more changes in genes related to DNA damage or repair, oxidative or metabolic stress, and proliferation than in genes related to heat shock. The 2 genes most significantly affected by magnetic-core treatment in vitro and in vivo were Cxcl10 (functional role, inflammation) and Cyp4a10 (functional role, oxidative stress). The Cxcl10 gene, which is related to inflammation, had an expression ratio of 17.51-fold and 4.30-fold compared with silica-coated magnetic-fluorescence nanoparticles, in vitro and in vivo, respectively. Also, the oxidative stress-related cyp4a10 gene had an expression ratio of 28.80-fold and 45.39-fold compared with silica-coated magnetic-fluorescence nanoparticles, in vitro and in vivo, respectively. The most highly affected of the 52 genes by magnetic-core treatment were Atm (related to DNA damage); Ccnd1, Ccnc, and Ccng1 (related to proliferation); and Cyp4a10 and Cyp4a14 (related to oxidative stress). Therefore, exposure of magnetic-core nanoparticles to liver tissue can cause severe oxidative stress or proliferation and even DNA damage.

In the case of metal-based nanomaterials for diagnostic and therapeutic applications in cancer and other diseases, safety standards for toxicologic effects should be established at the genetic and cellular levels. Therefore, we emphasize that clinical applications of nanomaterials can advance significantly through in vivo and in vitro research examining numerous gene expression profiles related to stress and toxicity pathways. The next step will be studies of the toxicologic effects of different shapes of nanomaterials, or long-term studies of these nanomaterials, measuring changes in the pattern of expression of multiple genes from severe cellular toxicity.

\section{CONCLUSION}

Cobalt ferrite magnetic nanomaterials have been proposed for medical application as a drug delivery system and have a substantial advantage over iron oxide nanomaterials. However, the use of cobalt ferrite for human medicine is still a distant goal because of severe genotoxicity and 
cytotoxicity. Therefore, we need to examine the effect of cobalt ferrite on the expression of critical genes related to oxidative stress, apoptosis, and cell damage, using a simple real-time PRC-based global gene expression assay.

In this study, in vitro and in vivo gene expression analysis indicated that cobalt ferrite magnetic-core nanoparticles were severely genotoxic to liver tissue, whereas magneticfluorescence nanoparticles had relatively safe levels of cytotoxicity and genotoxicity. Unlike previous studies investigating the level of gene expression from nanotoxicity, global gene expression profiles were simultaneously analyzed by simple and reliable real-time RT PCR, providing substantial information on the gene expression pattern induced by nanomaterials in vivo and in vitro. Multiple gene expression studies comparing magneticfluorescence and magnetic-core nanoparticles will offer important information for the application of nanomaterials to human studies for diagnostic or therapeutic purposes.

\section{ACKNOWLEDGMENTS}

This research was supported by grants from the Korea Healthcare Technology R\&D Project, Ministry for Health, Welfare and Family Affairs, Republic of Korea (A085136 and A100377); the Bio and Medical Technology Development Program of the National Research Foundation (NRF) funded by the Korean government (MEST) (2011-0019270); and the Brain Research Center of the 21st Century Frontier Research Program (2010K000817). No other potential conflict of interest relevant to this article was reported.

\section{REFERENCES}

1. De Jong WH, Borm PJ. Drug delivery and nanoparticles: applications and hazards. Int J Nanomedicine. 2008;3:133-149.

2. Haley B, Frenkel E. Nanoparticles for drug delivery in cancer treatment. Urol Oncol. 2008;26:57-64.

3. Hwang DW, Ko HY, Lee J, et al. A nucleolin-targeted multimodal nanoparticle imaging probe for tracking cancer cells using an aptamer. Nucl Med (Stuttg). 2010;51:98-105.
4. Hwang do W, Ko HY, Kim SK, Lee DS, Kim S. Development of a quadruple imaging modality by using nanoparticles. Chemistry. 2009;15:9387-9393.

5. Ko HY, Choi KJ, Lee CH, Kim S. A multimodal nanoparticle-based cancer imaging probe simultaneously targeting nucleolin, integrin $\alpha v \beta 3$ and tenascin-C proteins. Biomaterials. 2011;32:1130-1138.

6. Hussain SM, Hess KL, Gearhart JM, Geiss KT, Schlager JJ. In vitro toxicity of nanoparticles in BRL 3A rat liver cells. Toxicol In Vitro. 2005;19:975-983.

7. Miura N, Shinohara Y. Cytotoxic effect and apoptosis induction by silver nanoparticles in HeLa cells. Biochem Biophys Res Commun. 2009;390:733-737.

8. Braydich-Stolle L, Hussain S, Schlager JJ, Hofmann MC. In vitro cytotoxicity of nanoparticles in mammalian germline stem cells. Toxicol Sci. 2005;88:412-419.

9. Mahmood M, Casciano DA, Mocan T, et al. Cytotoxicity and biological effects of functional nanomaterials delivered to various cell lines. J Appl Toxicol. 2010;30:74-83.

10. Kawata K, Osawa M, Okabe S. In vitro toxicity of silver nanoparticles at noncytotoxic doses to HepG2 human hepatoma cells. Environ Sci Technol. 2009; 43:6046-6051.

11. Das GK, Chan PP, Teo A, et al. In vitro cytotoxicity evaluation of biomedical nanoparticles and their extracts. J Biomed Mater Res A. 2010;93:337-346.

12. Cho WS, Cho M, Jeong J, et al. Acute toxicity and pharmacokinetics of $13 \mathrm{~nm}-$ sized PEG-coated gold nanoparticles. Toxicol Appl Pharmacol. 2009;236:16-24.

13. Ponti J, Sabbioni E, Munaro B, et al. Genotoxicity and morphological transformation induced by cobalt nanoparticles and cobalt chloride: an in vitro study in Balb/3T3 mouse fibroblasts. Mutagenesis. 2009;24:439-445.

14. Kim JS, Yoon TJ, Yu KN, et al. Toxicity and tissue distribution of magnetic nanoparticles in mice. Toxicol Sci. 2006;89:338-347.

15. Yoon TJ, Yu KN, Kim E, et al. Specific targeting, cell sorting, and bioimaging with smart magnetic silica core-shell nanomaterials. Small. 2006;2:209-215.

16. Jeong JM, Hong MK, Chang YS, et al. Preparation of a promising angiogenesis PET imaging agent: ${ }^{68} \mathrm{Ga}$-labeled $\mathrm{c}(\mathrm{RGDyK})$-isothiocyanatobenzyl-1,4,7-triazacyclononane-1,4,7-triacetic acid and feasibility studies in mice. J Nucl Med. 2008;49:830-836.

17. Owens DE, Peppas NA. Opsonization, biodistribution, and pharmacokinetics of polymeric nanoparticles. Int J Pharm. 2006;307:93-102.

18. Fernández-Urrusuno R, Fattal E, Rodrigues MJ, Féger J, Bedossa P, Couvreur P. Effect of polymeric nanoparticle administration on the clearance activity of the mononuclear phagocyte system in mice. J Biomed Mater Res. 1996;31:401-408.

19. Fischer HC, Chan WC. Nanotoxicity: the growing need for in vivo study. Curr Opin Biotechnol. 2007;18:565-571.

20. Chen Z, Meng H, Xing G, et al. Acute toxicological effects of copper nanoparticles in vivo. Toxicol Lett. 2006;163:109-120.

21. Ziady AG, Gedeon CR, Muhammad O, et al. Minimal toxicity of stabilized compacted DNA nanoparticles in the murine lung. Mol Ther. 2003;8:948-956.

22. Yang H, Liu C, Yang D, Zhang H, Xi Z. Comparative study of cytotoxicity, oxidative stress and genotoxicity induced by four typical nanomaterials: the role of particle size, shape and composition. J Appl Toxicol. 2009;29:69-78.

23. Baldi G, Bonacchi D, Franchini MC, et al. Synthesis and coating of cobalt ferrite nanoparticles: a first step toward the obtainment of new magnetic nanocarriers. Langmuir. 2007;23:4026-4028. 